УДК: $532+532.6+517.97$

\title{
РАВНОВЕСНЫЙ ОБЪЕМ МАЛОЙ ЛЕЖАЩЕЙ КАПЛИ
}

\author{
(C) 2018 А. А. Сокуров \\ Институт прикладной математики и автоматизаџии КБНЦ РАН, \\ ул. Шортанова 89 A, 360000 КБР, г. Нальчик, Россия \\ e-mail: asokuroff@gmail.com \\ Поступила в редакцию 11.07.2018
}

\begin{abstract}
Аннотация. В настоящей работе рассматривается малая капля жидкости, которая лежит на горизонтальной гладкой поверхности в поле силы тяжести и пребывает в термодинамическом равновесии с собственным паром. С учетом размерной зависимости поверхностного натяжения получено уравнение, которое выступает в роли основного условия механического равновесия капли. Данное уравнение является аналогом уравнения Башфорта - Адамса, хорошо известного из математической теории равновесных капиллярных поверхностей. Исходя из аналога уравнения Башфорта - Адамса, получены системы нелинейных дифференциальных уравнений первого порядка, описывающие профиль капли. Найдена связь между координатами произвольной точки на поверхности капли и объемом заключенной жидкости. На вычислительном эксперименте смоделировано изменение линейных размеров капли с увеличением объема жидкости. Все указанные уравнения и формулы переходят в ранее известные, если в них параметр, отвечающий за размерный эффект поверхностного натяжения, приравнять к нулю.
\end{abstract}

Ключевые слова: лежащая капля, поверхностное натяжение, капиллярное давление, формула Лапласа, угол смачивания, капиллярная постоянная, равновесная капиллярная поверхность, размерная зависимость, длина Толмена, средняя кривизна, радиус кривизны, нанокапля.

DOI: https://doi.org/10.17308/kcmf.2018.20/583

\section{ВВЕДЕНИЕ}

На протяжении последних двух столетий равновесные состояния малых лежащих капель являются предметом интенсивных исследований в физике межфазных явлений [1]. Такой интерес, в первую очередь, обусловлен широтой их применения в методах по определению величины поверхностного натяжения и краевого угла смачивания, которые являются важнейшими термодинамическими характеристиками границ раздела фаз. Именно эти методы [2-4] считаются на практике самыми простыми, универсальными и одновременно самыми точными. Необходимо отметить, что основу большинства из них составляет использование математических моделей капель и различного рода количественных соотношений между координатами точки на поверхности капли и объемом заключенной жидкости $[5,6]$.

В настоящее время в связи с интенсивным развитием нанотехнологий все больше и больше внимания уделяется исследованию равновесных состояний очень малых объемов жидкости - нанокапель. Известно, что для малоразмерных капиллярных объ- ектов, нанокапель в частности, свойственны размерные эффекты. Суть размерных эффектов в данном случае заключается в зависимости какой-либо термодинамической величины от линейных размеров исследуемого капиллярного объекта. Это касается также поверхностного натяжения и краевого угла смачивания [7-11]. Поэтому при использовании нанокапель для изучения вопросов, связанных с размерной зависимостью поверхностного натяжения и краевого угла смачивания, обязательно возникнет необходимость в построении более адекватных математических моделей капель, нежели существующие на данный момент. Придется пересмотреть и количественные соотношения между координатами точки на поверхности капли и объемом. Попытке решения этих вопросов и посвящена данная статья.

\section{УРАВНЕНИЯ РАВНОВЕСНОЙ ПОВЕРХНОСТИ МАЛОЙ ЛЕЖАЩЕЙ КАПЛИ}

Рассмотрим малую каплю жидкости, лежащую на горизонтальной гладкой поверхности в поле силы 
тяжести и пребывающую в термодинамическом равновесии с собственным паром. Будем предполагать, что жидкость и материал, из которого сделана подложка, однородны. Это дает возможность полагать, что контактный угол смачивания $\theta$ постоянен. В работе [12] было показано, что равновесная поверхность такой капли обязательно будет поверхностью вращения с осью симметрии, перпендикулярной к подложке. По этой причине для того, чтобы вывести уравнение поверхности капли, достаточно будет вывести уравнение ее профиля в первом квадранте.

Свяжем с каплей систему декартовых координат $x O z$ таким образом, что начало координат совпадает с апексом капли, направление вертикальной оси $z$ совпадает с направлением вектора ускорения свободного падения, а горизонтальная ось $x$ параллельна плоскости подложки (см. рис. 1).

Основным условием механического равновесия любых двухфазных капиллярных систем в отсутствие других внешних полей кроме однородного гравитационного поля является баланс внутрифазовых гидростатический давлений и капиллярного давления, создаваемого искривленной границей раздела фаз. Применительно к нашей капле и выбранной системе координат оно запишется так

$$
2 \sigma H=\sigma\left(\frac{1}{R_{1}}+\frac{1}{R_{2}}\right)=p_{0}+\Delta \rho g z,
$$

где $\sigma$ - поверхностное натяжение на границе капли, $H$ - средняя кривизна поверхности, $R_{1}$ и $R_{2}$ - главные радиусы кривизны поверхности, $p_{0}$ - давление в апексе капли, $\Delta \rho-$ разность плотностей жидкой и газообразной фаз, $g$ - ускорение свободного падения. Уравнение (1) в теории капиллярности принято называть уравнением Лапласа.

Правая часть (1) представляет собой гидростатическое давление внутри капли, а левая - капил-

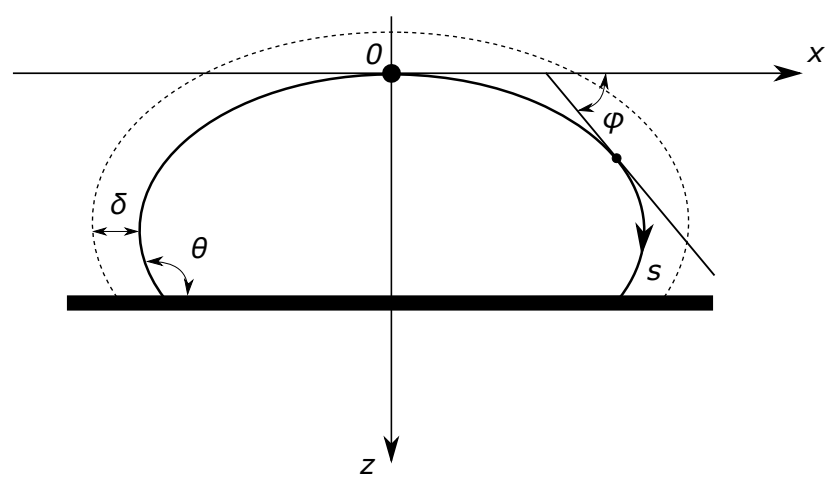

Рис. 1. Лежащая капля и связанная с ней система координат

[Fig. 1. Schematic of an axisymmetric liquid drop resting above a horizontal solid plane] лярное давление, рассчитываемое на основе формулы Лапласа. Для массивных капель в условиях термодинамического равновесия значение поверхностного натяжения $\sigma$ считается постоянной в каждой точке и зависит только от рода контактирующих сред. Такое допущение уже не приемлемо для очень малых капель, так как для поверхностного натяжения характерна размерная зависимость. Эта зависимость определяется следующей формулой [13]:

$$
\sigma=\frac{\sigma^{(\infty)}}{1+\delta\left(\frac{1}{R_{1}}+\frac{1}{R_{2}}\right)}
$$

где $\sigma^{(\infty)}$-поверхностное натяжение плоской поверхности, $\delta$ - неотрицательный параметр, характеризующий толщину межфазного слоя. Обычно в научной литературе он называются длиной Толмена и типичные его значения находятся в интервале 1-10 нM.

Перепишем условие механического равновесия (1) с учетом формулы (2)

$$
\frac{1}{R_{1}}+\frac{1}{R_{2}}=\frac{\lambda+\beta z}{1-\delta(\lambda+\beta z)},
$$

где для краткости записи введены обозначения: $\lambda=p_{0} / \sigma^{(\infty)}, \beta=\Delta \rho g / \sigma^{(\infty)}-$ капиллярная постоянная. Капиллярная постоянная $\beta$ наряду с капиллярной длиной $l_{c}=\beta^{-1 / 2}$ играет важную роль при оценке влияния гравитационного поля на форму капиллярных поверхностей. В частности, при $\beta=0$ очевидным решением уравнения (3) служит поверхность с постоянной положительной $(\delta \lambda<1)$ средней кривизной, которой является сфера. Соответственно, чем меньше $\beta$, тем ближе будет форма капли к сферической. Исходя из определения $\beta$, можно перечислить ситуации, когда реализуется условие $\beta \approx 0$ : плотность жидкости невелика; в невесомости $g=0$; поверхностное натяжение чрезвычайно велико. Последнее в особенности актуально для жидких металлов, например, для ртути. Однако на практике тривиальные решения в виде сферы не представляют особого интереса.

Для поверхности вращения имеем

$$
\frac{1}{R_{1}}=\frac{d \varphi}{d s}, \frac{1}{R_{2}}=\frac{\sin \varphi}{x},
$$

где $\varphi$ - угол наклона касательной к меридиональному сечению поверхности с положительным направлением оси $x, s$ - длина дуги. Поэтому уравнение (1) принимает вид

$$
\frac{d \varphi}{d s}+\frac{\sin \varphi}{x}=\frac{\lambda+\beta z}{1-\delta(\lambda+\beta z)}
$$


в котором его можно называть аналогом уравнения Башфорта-Адамса [13]. Дополнив (4) соотношениями, справедливыми для каждой гладкой кривой

$$
\left\{\begin{array}{l}
\frac{d x}{d s}=\cos \varphi, \\
\frac{d z}{d s}=\sin \varphi
\end{array}\right.
$$

сформулируем задачу Коши для определения равновесного профиля лежащей капли

$$
\begin{aligned}
& \int \frac{d x}{d s}=\cos \varphi, \\
& \frac{d z}{d s}=\sin \varphi \\
& \frac{d v}{d s}=\pi x^{2} \sin \varphi, \\
& \frac{d \varphi}{d s}=\frac{\lambda+\beta z}{1-\delta(\lambda+\beta z)}-\frac{\sin \varphi}{x}, \\
& x(s=0)=0, z(s=0)=0 \text {, } \\
& v(s=0)=0, \varphi(s=0)=0 .
\end{aligned}
$$

В систему (5) включено уравнение для объема $v$ жидкости, заключенного между апексом и произвольным уровнем $z$. От параметризации профиля по длине дуги можно перейти к параметризации по углу наклона касательной $\varphi$. Для этого достаточно разделить каждое уравнение в (5), кроме последнего, на $d \varphi / d s$ :

$$
\left\{\begin{array}{l}
\frac{d x}{d \varphi}=\frac{[1-\delta(\lambda+\beta z)] x \cos \varphi}{x(\lambda+\beta z)-[1-\delta(\lambda+\beta z)] \sin \varphi} \\
\frac{d z}{d \varphi}=\frac{[1-\delta(\lambda+\beta z)] x \sin \varphi}{x(\lambda+\beta z)-[1-\delta(\lambda+\beta z)] \sin \varphi} \\
\frac{d v}{d \varphi}=\frac{\pi[1-\delta(\lambda+\beta z)] x^{3} \sin \varphi}{x(\lambda+\beta z)-[1-\delta(\lambda+\beta z)] \sin \varphi}
\end{array}\right.
$$

$$
x(\varphi=0)=0, z(\varphi=0)=0, v(\varphi=0)=0 .
$$

На рис. 2 представлен профиль капли, построенный на основе численного решения задачи (7)-(8) при следующем наборе параметров: $\lambda=1$, $\delta=0.1, \beta=5$ и $\theta=135^{\circ}$, а на рис. 3 представлена соответствующая 3D-модель с различных ракурсов. В качестве метода нахождения приближенного решения использовался метод Рунге-Кутты 4-го порядка точности.

Для задачи (5)-(6) процесс численного интегрирования должен продолжаться до тех пор, пока не будет достигнуто пороговое значение либо для функции $\varphi(s)$, либо для $v(s)$.

\section{СВЯЗЬ ОБЬЕМА С КООРДИНАТАМИ ТОЧКИ}

Рассмотрим подробно уравнение для объема в системе (7). Проинтегрируем его по переменной $\varphi$ в пределах от 0 до некоторого $\varphi \in(0, \pi]$ :

$$
\begin{gathered}
v=\pi \int_{0}^{\varphi} x^{2} \frac{[1-\delta(\lambda+\beta z)] x \sin t}{x(\lambda+\beta z)-[1-\delta(\lambda+\beta z)] \sin t} d t= \\
=\pi \int_{0}^{\varphi} x^{2} d z=\pi\left(x^{2} z-2 \int_{0}^{\varphi} x z \frac{d x}{d \varphi}(t) d t\right)= \\
\pi\left(x^{2} z-2 \int_{0}^{\varphi} \frac{[1-\delta(\lambda+\beta z)] x^{2} z \cos t}{x(\lambda+\beta z)-[1-\delta(\lambda+\beta z)] \sin t} d t\right) .
\end{gathered}
$$

Преобразуем в последней скобке подынтегральную функцию. Для этого заметим, что

$$
\begin{aligned}
& \frac{[1-\delta(\lambda+\beta z)] x^{2} z \cos \varphi}{x(\lambda+\beta z)-[1-\delta(\lambda+\beta z)] \sin \varphi}= \\
= & \frac{1}{\beta} \frac{d}{d \varphi}\left(x \sin \varphi-\frac{\lambda x^{2}}{2}\right)- \\
- & \frac{\delta}{\beta} \frac{x^{2}(\lambda+\beta z)^{2} \cos \varphi}{x(\lambda+\beta z)-[1-\delta(\lambda+\beta z)] \sin \varphi},
\end{aligned}
$$

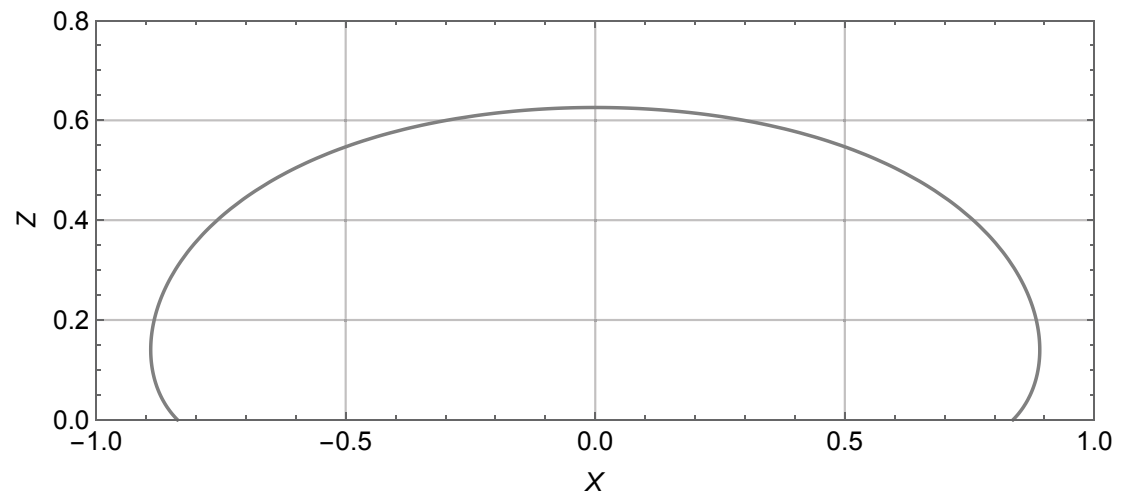

Рис. 2. Профиль лежащей капли при $\lambda=1, \delta=0.1, \beta=5, \theta=135^{\circ}$

[Fig. 2. Sessile drop profile for $\lambda=1, \delta=0.1, \beta=5, \theta=135^{\circ}$ ] 

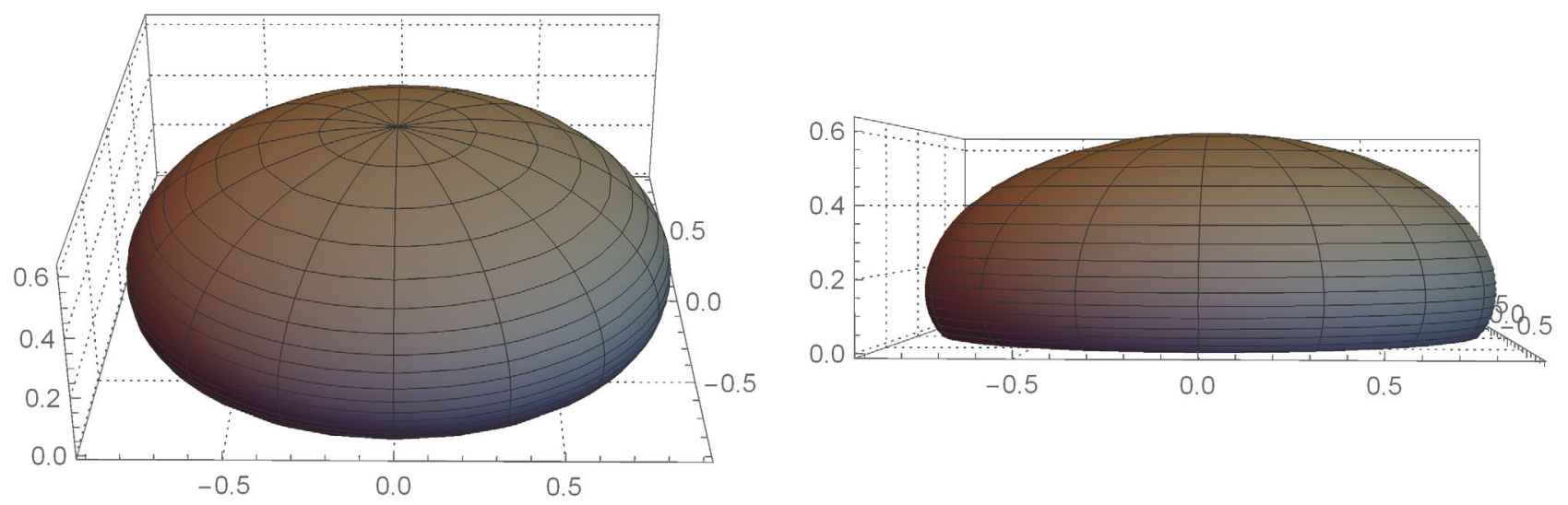

Рис. 3. Трехмерная модель лежащей капли при $\lambda=1, \delta=0.1, \beta=5, \theta=135^{\circ}$

[Fig. 3. 3D model of the sessile drop for $\lambda=1, \delta=0.1, \beta=5, \theta=135^{\circ}$ ]

с учетом чего (9) перепишется в виде следующего тождества

$$
\begin{gathered}
v=\pi x^{2} z-\frac{2 \pi x}{\beta}\left(\sin \varphi-\frac{\lambda x}{2}\right)+ \\
+\frac{2 \pi \delta}{\beta} \int_{0}^{\varphi} \frac{x^{2}(\lambda+\beta z)^{2} \cos t}{x(\lambda+\beta z)-[1-\delta(\lambda+\beta z)] \sin t} d t .
\end{gathered}
$$

При $\delta=0$ соотношение (10) принимает очень простую форму

$$
v=\pi x^{2} z-\frac{2 \pi x}{\beta}\left(\sin \varphi-\frac{\lambda x}{2}\right) .
$$

Однако при $\delta \neq 0$ интеграл, содержащийся в (10), уже не удается выразить в явном виде через элементарные функции. Тем не менее, (10) может оказаться полезной при получении различных аппроксимационных формул и оценок. Например, при достаточно малых $\varphi$ по формуле трапеции

$$
\begin{gathered}
\int_{0}^{\varphi} \frac{x^{2}(\lambda+\beta z)^{2} \cos t}{x(\lambda+\beta z)-[1-\delta(\lambda+\beta z)] \sin t} d t= \\
=\frac{x^{2}(\lambda+\beta z)^{2} \cos \varphi}{x(\lambda+\beta z)-[1-\delta(\lambda+\beta z)] \sin \varphi} \frac{\varphi}{2}+O\left(\varphi^{2}\right) .
\end{gathered}
$$

Следовательно

$$
\begin{gathered}
v \approx \pi x^{2} z-\frac{2 \pi x}{\beta}\left(\sin \varphi-\frac{\lambda x}{2}\right)+ \\
+\frac{\pi \delta \varphi}{\beta} \frac{x^{2}(\lambda+\beta z)^{2} \cos \varphi}{x(\lambda+\beta z)-[1-\delta(\lambda+\beta z)] \sin \varphi} .
\end{gathered}
$$

Как показывают численные расчеты, в окрестности апекса капли формула (11) дает удовлетворительные результаты, и относительная погрешность составляет порядка нескольких процентов.

\section{ОЦЕНКИ И АСИМПТОТИКА ДЛЯ РАВНОВЕСНОГО ОБЪЕМА}

Несмотря на то, что значение объема $v$ не удается явно выразить через координаты точки и угол наклона касательной, как это имеет место при $\delta=0$, его можно оценить сверху и снизу. Для этого рассмотрим аналог уравнения Башфорта-Адамса (4), обе части которого умножим на $x d x / d \varphi$. При этом левая часть преобразуется следующим образом

$$
\begin{gathered}
x \frac{d x}{d \varphi}\left(\frac{d \varphi}{d s}+\frac{\sin \varphi}{x}\right)=x \frac{d x}{d s}+\frac{d x}{d \varphi} \sin \varphi= \\
=x \cos \varphi+\frac{d x}{d \varphi} \sin \varphi=\frac{d}{d \varphi}(x \sin \varphi) .
\end{gathered}
$$

Получим

$$
\frac{d}{d \varphi}(x \sin \varphi)=\frac{\lambda+\beta z}{1-\delta(\lambda+\beta z)} x \frac{d x}{d \varphi} .
$$

Проинтегрируем (12) по переменной $\varphi$ в пределах от 0 до некоторого $\varphi \in(0, \pi / 2]$ :

$$
x \sin \varphi=\int_{0}^{\varphi} \frac{\lambda+\beta z}{1-\delta(\lambda+\beta z)} \frac{d x}{d \varphi}(t) x d t .
$$

Далее, оценим (13), учитывая, что подынтегральная функция везде неотрицательна и вдоль профиля капли $z(\varphi)$ монотонно возрастает. С одной стороны

$$
\begin{gathered}
\frac{1}{1-\delta \lambda} \int_{0}^{\varphi}(\lambda+\beta z) x \frac{d x}{d \varphi}(t) d t \leq x \sin \varphi= \\
=\int_{0}^{\varphi} \frac{\lambda+\beta z}{1-\delta(\lambda+\beta z)} \frac{d x}{d \varphi}(t) x d t,
\end{gathered}
$$

а с другой 


$$
\begin{aligned}
& x \sin \varphi=\int_{0}^{\varphi} \frac{\lambda+\beta z}{1-\delta(\lambda+\beta z)} \frac{d x}{d \varphi}(t) x d t \leq \\
& \leq \frac{1}{1-\delta(\lambda+\beta z)} \int_{0}^{\varphi}(\lambda+\beta z) x \frac{d x}{d \varphi}(t) d t .
\end{aligned}
$$

Неравенства (14) нестрогие и знак равенства в них достигается только в том случае, если $\delta=0$. Из (14a) следует

$$
\int_{0}^{\varphi} z x \frac{d x}{d \varphi}(t) d t \leq \frac{1}{\beta}\left(x(1-\delta \lambda) \sin \varphi-\frac{\lambda x^{2}}{2}\right),
$$

а из $(14 b)$

$\frac{1}{\beta}\left(x(1-\delta(\lambda+\beta z)) \sin \varphi-\frac{\lambda x^{2}}{2}\right) \leq \int_{0}^{\varphi} z x \frac{d x}{d \varphi}(t) d t$.

Так как капля по предположению представляет собой тело вращения, то

$$
v=\pi \int_{0}^{\varphi} x^{2} d z=\pi\left(x^{2} z-2 \int_{0}^{\varphi} x z \frac{d x}{d \varphi}(t) d t\right) .
$$

Поэтому, оценивая с помощью (15) интеграл в скобке (16), окончательной приходим к двойному неравенству

$$
v^{-}=\pi x^{2} z-\frac{2 \pi}{\beta}\left(x(1-\delta \lambda) \sin \varphi-\frac{\lambda x^{2}}{2}\right) \leq v,(17 a)
$$$$
v \leq \pi x^{2} z-\frac{2 \pi}{\beta}\left(x(1-\delta(\lambda+\beta z)) \sin \varphi-\frac{\lambda x^{2}}{2}\right)=v^{+} .
$$

Таким образом, значение объема любой капли при $\delta \neq 0$ и $\varphi \in(0, \pi / 2]$ всегда заключено между числами $v^{-}$и $v^{+}$. Исходя из этого, с абсолютной погрешностью не превосходящей $\varepsilon$

$$
\varepsilon=v^{+}-v^{-}=2 \pi x z \delta \sin \varphi,
$$

за $v$ можно принять любое число из отрезка $\left[v^{-}, v^{+}\right]$. Видно, что погрешность зависит от степени близости точки к апексу капли, а также от малости $\delta$ и $\varphi$.
Проанализируем теперь характер изменения линейных размеров капли при постепенном увеличении ее объема. Увеличение объема сопровождается увеличением площади области контакта жидкости с твердой подложкой и увеличением центральной высоты $z_{\max }=z(x=0)$. Однако рост $z_{\max }$ при росте $v$ не может продолжаться бесконечно. Установлено $[15,16]$, что для каждой фиксированной трехфазной системы «капля - пар - твердая подложка» существует некоторое критическое значение объема жидкости $v^{*}$, после достижения которого увеличение центральной высоты капли должно прекратиться. При значениях $v>v^{*}$ центральная высота $z_{\max }$ остается постоянной. Дальнейшее увеличение объема, как показывают опыты, приводит к тому, что растет площадь области контакта жидкости с подложкой и усиливается «горизонтальность» верхушки капли. В результате капля приобретает форму цилиндра, радиус основания которого намного превосходит высоту.

Описанное выше можно смоделировать на вычислительном эксперименте. Для этого достаточно заметить, что изменениям объема капли соответствуют изменения значений лишь одного параметра $\lambda$ в системе уравнений (7). Параметры $\delta$ и $\beta$, отвечающие за физические свойства трехфазной системы, должны оставаться неизменными. На рис. 3 построены профили капель различных объемов: сплошная линия соответствует $\lambda=0.5$, точечная $-\lambda=0.25$, пунктирная $-\lambda=0.1$. Во всех случаях $\theta=\pi / 2$.

Видно, что меньшим значениям $\lambda$ соответствуют бо́льшие значения объема. Исходя из уравнения Лапласа (3), можно установить смысл параметра $\lambda$. В начале координат оно принимает вид

$$
\frac{2}{R_{0}}=\frac{\lambda}{1-\delta \lambda}
$$

где $R_{0}$ - радиус кривизны профиля капли в апексе. Таким образом, заключаем, что при фиксированном

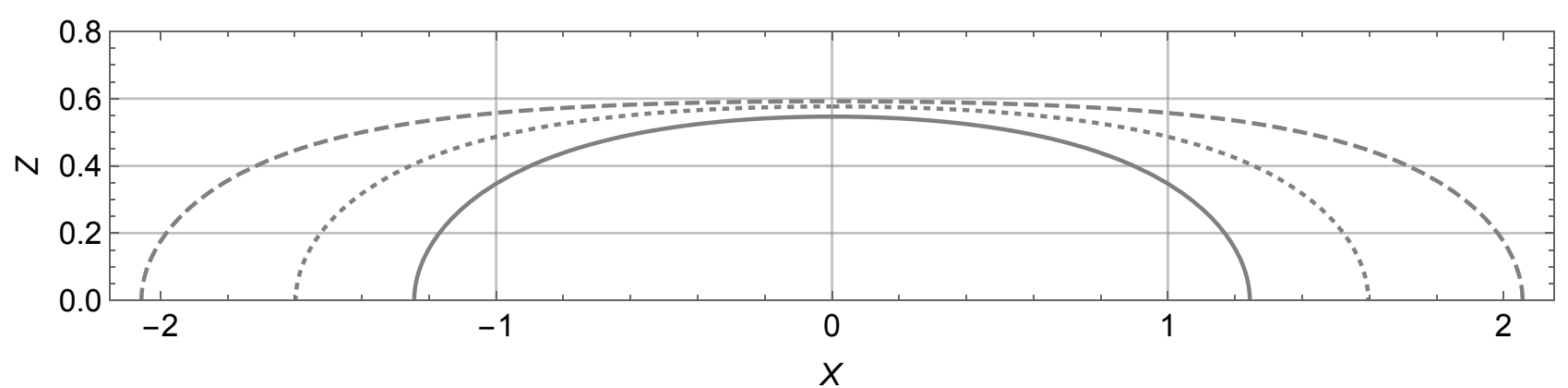

Рис. 4. Изменение формы лежащей капли при изменении объема

[Fig. 4. Effect of volume on the sessile drop profile] 
$\delta$ параметр $\lambda$ отвечает за кривизну верхушки капли. Так как правая часть (18) является возрастающей функцией от $\lambda$, то уменьшение $\lambda$ одновременно с увеличением объема приводит к уменьшению искривленности профиля капли в окрестности апекса, и капля становится все больше похожей на диск.

Из всего сказанного следует, что при малых $\lambda$ должно иметь место следующее асимптотическое равенство, вытекающее из «цилиндричности» капли

$$
v \approx \pi x^{2} z .
$$

Действительно, разделим обе части неравенств (17) на $\pi x^{2} z$

$$
\begin{gathered}
1-\frac{2}{\beta z}\left((1-\delta \lambda) \frac{\sin \varphi}{x}-\frac{\lambda}{2}\right) \leq \frac{v}{\pi x^{2} z}, \\
\frac{v}{\pi x^{2} z} \leq 1-\frac{2}{\beta z}\left((1-\delta(\lambda+\beta z)) \frac{\sin \varphi}{x}-\frac{\lambda}{2}\right) .
\end{gathered}
$$

Азимутальная кривизна $k_{m}$ поверхности капли, фигурирующее в (20)

$$
k_{m}=\frac{\sin \varphi}{x}
$$

при движении вдоль профиля капля монотонно возрастает [17] и достигает свое максимальное значение в точке $\varphi=\pi / 2$ :

$$
k_{m} \leq \frac{1}{x_{r}}, \varphi \in(0, \pi / 2],
$$

где $x_{r}$ - радиус капли. Для широких капель $x_{r}$ достигает больших значений, следовательно

$$
k_{m}(\varphi) \leq k_{m}\left(\frac{\pi}{2}\right) \ll 1, \varphi \in(0, \pi / 2]
$$

Получается, что при уменьшении $\lambda$ выражения, стоящие в скобках (20) становятся пренебрежимо малыми. А учитывая, что значения $z$ ограничены, приходим к требуемому асимптотическому соотношению (19):

$$
\frac{v}{\pi x^{2} z} \rightarrow 1 \text { при } \lambda \rightarrow 0 .
$$

\section{ЗАКЛЮЧЕНИЕ}

Рассмотрена малая капля жидкости, которая лежит на горизонтальной гладкой поверхности в поле силы тяжести и пребывает в термодинамическом равновесии с собственным паром. С учетом размерной зависимости поверхностного натяжения получено уравнение, которое выступает в роли основного условия механического равновесия поверхности капли. Данное уравнение явля- ется аналогом уравнения Башфорта-Адамса, хорошо известного из математической теории равновесных капиллярных поверхностей. Исходя из аналога уравнения Башфорта-Адамса, получены системы нелинейных дифференциальных уравнений первого порядка, описывающие профиль капли. В первом случае в качестве переменной параметризации выступает длина дуги, а во втором - угол наклона касательной. Задача Коши для полученных систем дифференциальных уравнений имеет аналитическое решение только в тривиальном случае, когда параметр, связанный с напряженностью гравитационного поля, равен нулю. Это решение представляет собой сегмент сферы. В остальных случаях выписать точное решение в явной форме не представляется возможным, в связи с чем возникает необходимость в использовании вычислительных методов и комплексов программ. Установлено, что в качестве эффективного численного метода нахождения приближенного решения сформулированных задач может быть использован метод Рунге-Кутты четвертого порядка точности. Найдено интегральное соотношение между координатами произвольной точки на поверхности капли и объемом заключенной жидкости. В общем случае данное соотношение не выражается в явном виде через элементарные функции. Поэтому для него получены аппроксимационная формула и асимптотическое равенство, а также оценки с избытком и недостатком (с указанием точности). На вычислительном эксперименте смоделировано изменение линейных размеров капли с увеличением объема жидкости. Все указанные уравнения и формулы переходят в ранее известные, если параметр, отвечающий за размерный эффект поверхностного натяжения, приравнять к нулю. Полученные в работе результаты могут найти применение при разработке и совершенствовании методик по определению поверхностного натяжения и при изучении капиллярных явлений второго рода.

\section{СПИСОК ЛИТЕРАТУРЫ}

1. Матюхин С. И., Фроленков К. Ю. // Конденсированные среды и межфазные границы, 2013, т. 15, № 3, c. 292-304.

2. Del Río O. I., Neumann A. W. // Journal of Colloid and Interface Science, 1997, vol. 196, № 2, pp. 136-147. DOI: https://doi.org/10.1006/jcis.1997.5214

3. Rotenberg Y., Boruvka L., Neumann A. W. // Journal of Colloid and Interface Science, 1983, vol. 93, № 1, pp. 169183. DOI: https://doi.org/10.1016/0021-9797(83)90396-X

4. Aurélien F. S., et al. // Colloids and Surfaces A: Physicochemical and Engineering Aspects, 2010, vol. 364, 
№ 1-3, pp. 72-81. DOI: https://doi.org/10.1016/j.colsurfa.2010.04.040

5. Faour G., et al. // Journal of Colloid and Interface Science, 1996, vol. 181, № 1, pp. 385-392. DOI: https://doi. org/10.1006/jcis.1996.0395

6. Русанов А. И., Прохоров В. А. Межфазная тензиометрия, СПб.: Химия, 1994, 400 с.

7. Tolman R. C. // The Journal of Chemical Physics, 1949, vol. 17, № 3, pp. 333-337. DOI: 10.1063/ 1.1747247

8. Рехвиашвили С. Ш., Киштикова Е. В. // ЖТФ, 2011, т. 81, № 1, с. 148-152.

9. Рехвиашвили С. Ш., Киштикова Е. В. // Физикохимия поверхности и защчита материалов, 2014, т. 50, № 1, c. 3-7.

10. Kalová J., Mareš R. // International Journal of Thermophysics, 2015, vol. 36, № 10-11, pp. 2862-2868. DOI: https://doi.org/10.1007/s1076

11. Burian S. // Physical review E, 2017, vol. 95, № 6, p. 062801 . DOI: https://doi.org/10.1103/ PhysRevE.95.062801

12. Wente H. C. // Pacifc J. Math., 1980, vol. 88, № 2, pp. 387-397.

13. Сокуров А. А., Рехвиашвили С. Ш. // Конденсированные среды и межфазные границыл, 2013, т. 15, № 2, c. $173-178$.

14. Bashforth F., Adams J. C., An Attempt to Test the Theories of Capillary Action by Comparing the Theoretical and Measured Forms of Drops of Fluid. University Press, Cambridge, 1883,158 p.

15. Канчукоев В. 3. // ЖТФ, 2004, т. 3, № 2, с. 12 16.

16. Марков И. И. и др. // Вестник Северо-Кавказского государственного технического университета, 2009, т. 19, № 2, c. 51-58.

17. Finn R. Equilibrium Capillary Surfaces. New York, Springer, 1986, 284 p. DOI: https://doi.org/10.1007/978-14613-8584-4

\title{
EQUILIBRIUM VOLUME OF A SMALL SESSILE DROP
}

\author{
(C) 2018 A. A. Sokurov \\ Institute of Applied Mathematics and Automation of Kabardin-Balkar Scientific Centre of RAS, 89 A Shortanova str., \\ 360000 Nalchik, Russia \\ e-mail: asokuroff@gmail.com
}

Received 11.07.2018

\begin{abstract}
In the current paper we consider a small liquid drop resting on a horizontal smooth surface with the effect of gravity when it is in thermodynamic equilibrium with its own vapor. An equation that expresses the main condition for the mechanical equilibrium of the droplet surface is obtained taking into account the size dependence of the surface tension. This equation is an analog of the Bashforth - Adams equation that is well known from the mathematical theory of equilibrium capillary surfaces. Based on the analog of the Bashforth - Adams equation systems of nonlinear first-order differential equations describing the drop profile are obtained. The Cauchy problem for the resulting systems of differential equations has an analytical solution only in the trivial case when the parameter associated with the gravitational field strength equals zero. In other cases it is not possible to find an exact solution which makes it necessary to use numerical methods and software complexes. It is established that the Runge - Kutta fourth order method may be used as an effective numerical method for finding the approximate solution of the formulated problems. An integral relation between the coordinates of an arbitrary point on the droplet surface and the volume of the enclosed liquid is found. In the general case this relation is not expressed in terms of elementary functions. For this reason an approximation formula, an asymptotic equality and estimates (indications of the accuracy are given) are obtained for it. The computational experiment on the effect of the volume of a liquid on the droplet shape is presented. All equations and formulas go over to the earlier known if the parameter responsible for the size effect equals zero. The results obtained in the work may find application in the development of methods for the determination of surface tension and in studying the second kind capillary phenomena.
\end{abstract}

Keywords: sessile drop, surface tension, capillary pressure, Laplace equation, contact angle, capillary constant, equilibrium capillary surface, size dependence, Tolman length, mean curvature, radius of curvature, nano-droplet.

DOI: https://doi.org/10.17308/kcmf.2018.20/583 


\section{REFERENCES}

1. Matyukhin S. I., Frolenkov K. Yu. Condensed Matter and Interphases, 2013, vol. 15, no. 3, pp. 292-304. Available at: http://www.kcmf.vsu.ru/resources/t_15_3_2013_012.pdf (in Russ.)

2. Del Río O. I., Neumann A. W. Journal of Colloid and Interface Science, 1997, vol. 196, no. 2, pp. 136-147. DOI: 10.1006/jcis. 1997.5214

3. Rotenberg Y., Boruvka L., Neumann A. W. Journal of Colloid and Interface Science, 1983, vol. 93, no. 1, pp. 169-183. DOI: 10.1016/0021-9797(83)90396-X

4. Aurélien F. S., et al. Colloids and Surfaces A: Physicochemical and Engineering Aspects, 2010, vol. 364, no. 1-3, pp. 72-81. DOI: 10.1016/j.colsurfa.2010.04.040

5. Faour G., et al. Journal of Colloid and Interface Science, 1996, vol. 181, no. 2, pp. 385-392. DOI: 10.1006/ jcis. 1996.0395

6. Rusanov A. I., Prokhorov V. A. Mezhfaznaya tenziometriya [Interfacial Tensiometry]. Saint Petersburg, Khimiya Publ., 1994, 400 p. (in Russ.)

7. Tolman R. C. The Journal of Chemical Physics, 1949, vol. 17, no. 3, pp. 333-337. DOI: 10.1063/1.1747247

8. Rekhviashvili S. Sh., Kishtikova E. V. Zhurnal tekhnicheskoj fiziki [Technical Physics. The Russian Journal of Applied Physics], 2011, vol. 81, no. 1, pp. 148-152. Available at: http://journals.ioffe.ru/articles/viewPDF/10213 (in Russ.)

9. Rekhviashvili S. Sh., Kishtikova E. V. Fizikokhimiya poverkhnosti $i$ zashhita materialov [Protection of Metals and Physical Chemistry of Surfaces], 2014, vol. 50, no. 1, pp. 3-7. DOI: 10.7868/S0044185614010112 (in Russ.)

10. Kalová J., Mareš R. International Journal of Thermophysics, 2015, vol. 36, no. 10-11, pp. 2862-2868. DOI: https://doi.org/10.1007/s1076

11. Burian S. Physical review E, 2017, vol. 95, no. 6, p. 062801. DOI: 10.1103/PhysRevE.95.062801

12. Wente H. C. Pacifc J. Math., 1980, vol. 88, no. 2, pp. 387-397.

13. Sokurov A. A., Rekhviashvili S. Sh. Condensed Matter and Interphases, 2013, vol. 15, no. 2, pp. 173-178. Available at: http://www.kcmf.vsu.ru/resources/t_15_2_ 2013_014.pdf (in Russ.)

14. Bashforth F., Adams J. C. An Attempt to Test the Theories of Capillary Action by Comparing the Theoretical and Measured Forms of Drops of Fluid, University Press, Cambridge, 1883, $158 \mathrm{p}$.

15. Kanchukoev V. Z. Pis'ma v Zhurnal tekhnicheskoj fiziki [Technical Physics Letters], 2004, vol. 3, no. 2, pp. 12-16. Available at: http://journals.ioffe.ru/articles/ viewPDF/11286 (in Russ.)

16. Markov I. I., et al. Vestnik Severo-Kavkazskogo gosudarstvennogo tekhnicheskogo universiteta [Journal Newsletter of North-Caucasus State Technical University], 2009, vol. 19, no. 2, pp. 51-58. Available at: https://elibrary. $\mathrm{ru} / \mathrm{item} . \mathrm{asp}$ ?id=12833518 (in Russ.)

17. Finn R. Equilibrium Capillary Surfaces. New York, Springer, 1986, 284 p. DOI: 10.1007/978-1-4613-8584-4

Aslan A. Sokurov - Junior Researcher, Institute of Applied Mathematics and Automation of Kabardin-Balkar Scientific Centre of RAS; tel.: +7(965) 4958002, e-mail: asokuroff@gmail.com 